that reduced LC ADAM17 activity in two lupus models contributed to their photosensitivity. Non-lesional human SLE skin also showed evidence of a dysfunctional LC-keratinocyte axis; however, what causes LC dysfunction is not known. Here we test the hypothesis that IFN-I in the skin contributes to LC ADAM17 dysfunction and thus photosensitivity.

Methods To assess IFN-I gene signature, microarray of nonlesional skin from human cutaneous LE and RNA sequencing of whole skin from lupus mouse models were performed. To quantify human and murine LC ADAM17 activity and expression, in vitro and ex vivo flow cytometric-based assays were conducted LCs. To assay photosensitivity, readouts of skin inflammation and cellular infiltrate were measured and characterized. Results We show that non-lesional skin from human cutaneous LE and photosensitive MRL/lpr and B6.Sle1yaa mice all share IFN-I signatures and that IFN-I is sufficient to reduce human and murine LC ADAM17 activity independently of surface ADAM17 levels. IFN-I induced LC ADAM17 activity defects were abrogated with tofaticinib, a JAK kinase inhibitor approved for rheumatoid arthritis and other rheumatologic diseases. We further show that anti-IFNAR1 treatment prior to UVR exposure in lupus models restores LC ADAM17 activity and limits photosensitivity.

Conclusions Together, our results suggest a model whereby the elevated IFN-I in non-lesional skin contributes to photosensitivity at least in part by causing LC ADAM17 dysfunction. The corollary is that anti-IFNAR has beneficial effects at least in part by correcting LC ADAM17 dysfunction.

Acknowledgements This work was supported by National Institutes of Health R01AI079178 (TTL), the Lupus Research Alliance (TTL), the St. Giles Foundation (TTL) and A $\Omega$ A Carolyn L. Kuckein Student Research Fellowship (TML).

\section{CAUSES OF DEATH AMONG POPULATIONS WITH SYSTEMIC LUPUS ERYTHEMATOSUS BY SEX, RACE AND ETHNICITY}

${ }^{1}$ Milena A Gianfrancesco*, 'Tiffany Taylor, 'Christine Anastasiou, ${ }^{1}$ Stephanie Rush, ${ }^{1}$ Laura Trupin, ${ }^{1}$ Maria Dall'Era, ${ }^{1}$ Patricia Katz, ${ }^{2}$ Kamil E Barbour, ${ }^{1}$ Jinoos Yazdany. ${ }^{1}$ Division of Rheumatology, School of Medicine, University of California, San Francisco, San Francisco, $C A ;{ }^{2}$ Division of Population Health, Centers for Disease Control and Prevention, Atlanta, Georgia

\subsection{6/lupus-2021-lupus21century.6}

Background Data indicate that minority populations with systemic lupus erythematosus (SLE) are at higher risk of developing disease and have more severe outcomes, including mortality. However, whether specific causes of death vary by race and ethnicity has largely been unexplored, particularly for Asians and Hispanics.

Methods The California Lupus Surveillance Project identified potential SLE cases using community rheumatology and nephrology clinics, community hospitals, and integrated healthcare systems among individuals who were residents of San Francisco County, CA during January 1, 2007 - December 31, 2009. Cases were matched to the 2007-2017 National Death Index (NDI) data, which included the underlying cause of death for each individual. Chi-squared tests were used to examine differences in underlying cause of death by race (white, Black, Asian), ethnicity (Hispanic, non-Hispanic), and sex. Age-standardized mortality ratios (SMRs) between SLE patients and the general San Francisco county population were calculated for the leading cause of death, and estimated
Abstract 202 Table 1 Standardized mortality ratios of cardiovascular disease (CVD) in SLE patients compared to the general San Francisco county population, age-standardized, 2007-2017

\begin{tabular}{lllll}
\hline & \multirow{2}{*}{$\begin{array}{l}\text { Total SLE } \\
\text { Population }\end{array}$} & \multicolumn{3}{l}{ CVD as Underlying Cause of Death } \\
\cline { 3 - 5 } & \multicolumn{5}{c}{$\begin{array}{l}\text { Observed CVD } \\
\text { Deaths in CLSP }\end{array}$} & $\begin{array}{l}\text { Expected } \\
\text { CVD Deaths }\end{array}$ & SMR $(95 \% \mathrm{CI})$ \\
\hline $\begin{array}{l}\text { Overall } \\
\text { Race }\end{array}$ & 809 & 45 & 12.4 & $3.63(2.65,4.86)$ \\
White & 311 & 13 & 5.3 & $2.43(1.29,4.16)$ \\
$\begin{array}{l}\text { Black } \\
\text { Asian }\end{array}$ & 164 & 16 & 5.5 & $2.89(1.65,4.70)$ \\
Ethnicity & 294 & 14 & 3.7 & $3.83(2.09,6.42)$ \\
Hispanic & 123 & 7 & 1.1 & $6.45(2.59,13.29)$ \\
Non-Hispanic & 604 & 36 & 10.6 & $3.39(2.37,4.69)$ \\
Sex & & & & \\
Female & 728 & 40 & 8.6 & $4.65(3.32,6.34)$ \\
Male & 81 & 5 & 1.4 & $3.48(1.13,8.12)$ \\
\hline
\end{tabular}

observed versus expected deaths by sex, race, and Hispanic/ Latino ethnicity.

Results During the study period, 135 deaths related to SLE were identified $(n=809)$. The top underlying cause of death overall (33\%) and across all racial and ethnic groups was cardiovascular disease (CVD). Other top causes of death included rheumatic disease $(18 \%)$ and hematological/oncological conditions (18\%) overall, and across all racial groups. Analyses examining any cause of death indicated that rheumatic disease was more commonly indicated among white (50\%) and Asian (46\%) patients as compared to Black (27\%) patients with SLE. In comparison to the general population of San Francisco County, CVD as the underlying cause of death was over three times higher among individuals with SLE $(\mathrm{SMR}=3.63)$ (table 1). CVD deaths for those with SLE were nearly three times higher for Black, approximately four times higher for Asian, and over six times higher for Hispanic/Latino individuals. CVD deaths were also elevated for females $(S M R=4.7)$ and males $(\mathrm{SMR}=3.5)$ with SLE compared to the general population.

Conclusions Our results show that CVD is the leading underlying cause of death among SLE patients across various racial and ethnic groups, and that rheumatic disease may be less likely to be listed as a cause of death among Black patients with SLE. Further, Asian and Hispanic/Latino SLE patients experience a disproportionate burden of CVD mortality compared with the general population.

Acknowledgements This work was support by grants from the Lupus Foundation of America (TT) and the Rheumatology Research Foundation (TT).

\section{NON-LESIONAL AND LESIONAL LUPUS SKIN SHARE INFLAMMATORY PHENOTYPES THAT DRIVE ACTIVATION OF CD16+ DCS}

${ }^{1}$ Allison C Billi, ${ }^{2}$ Feiyang Ma, ${ }^{1}$ Olesya Plazyo, ${ }^{1}$ Rachael Wasikowski, ${ }^{1,3}$ Mehrnaz GharaeeKermani, ${ }^{3}$ Amy Hurst, ${ }^{1}$ Craig J Dobry, 'Lam C Tsoi, ${ }^{1} J o h a n n$ E Gudjonsson, ${ }^{1,3}$ J Michelle Kahlenberg*. ${ }^{1}$ Department of Dermatology, University of Michigan, Ann Arbor, MI, USA; ${ }^{2}$ Departmnet of Molecular, Cell, and Developmental Biology, University of California Los Angeles, Los Angeles, CA, USA; ${ }^{3}$ Division of Rheumatology, Dept of Internal Medicine, University of Michigan, Ann Arbor, MI, USA

10.1136/lupus-2021-lupus21 century.7 